\title{
Effects of prostaglandin analog therapy on the ocular surface of glaucoma patients
}

\author{
Michael B Horsley \\ Malik Y Kahook
}

Rocky Mountain Lions Eye Institute, Department of Ophthalmology, University of Colorado Denver, Aurora, CO, USA
Correspondence: MalikY Kahook Rocky Mountain Lions Eye Institute, University of Colorado Denver, I 675 N. Ursula Street, Mail Stop F-73 I, Aurora, CO 80045, USA

Tel +l 7208482500

$\mathrm{Fax}+\mid 7208485014$
Purpose: To quantify changes in tear break-up time (TBUT), corneal staining and ocular surface disease index (OSDI) in glaucoma patients after switching therapy from latanoprost with $0.02 \%$ benzalkonium chloride (BAK) to travoprost with $s o f \mathrm{Zia}^{\mathrm{TM}}$.

Methods: Prospective consecutive case series evaluating patients before and 8 weeks after switching from latanoprost with BAK to travoprost with sof $\mathrm{Zia}^{\text {тм }}$ in patients with baseline TBUT less than 6 seconds.

Results: Forty eyes of 20 consecutive patients using latanoprost with BAK were switched to travoprost with sofZia ${ }^{\mathrm{TM}}$. Mean TBUT prior to starting travoprost was $2.02 \pm 0.71$ seconds and increased to $6.34 \pm 1.31$ seconds 8 weeks after the switch $(\mathrm{p}<0.001)$. Mean inferior corneal staining scores decreased from $2.40 \pm 0.87$ to $1.38 \pm 0.59(\mathrm{p}<0.001)$. Mean OSDI scores decreased from $26.31 \pm 8.25$ to $16.56 \pm 6.19(\mathrm{p}<0.001)$.

Discussion: This report focuses on the status of the ocular surface, as documented by TBUT, corneal staining and OSDI, in patients switched from latanoprost with BAK to travoprost without BAK. The switch resulted in a statistically significant increase in TBUT and decreases in corneal staining and OSDI in patients with low baseline TBUT values.

Conclusion: BAK, a common preservative for glaucoma drops, may increase OSD by disrupting the tear film and increasing conjunctival inflammation. In this study, a change from a BAK-preserved prostaglandin analog (PGA) to a non-BAK-preserved PGA resulted in a measurable improvement of TBUT, corneal staining and OSDI. Further studies are needed to better understand the impact of BAK-preserved medications on the ocular surface.

Keywords: ocular surface, glaucoma, benzalkonium chloride, prostaglandin analog

The US Food and Drug Administration requires manufacturers of multi-dose topical ophthalmic medications to include preservative systems against microbial contamination. Clinicians have long understood that, along with the benefits of safety in prolonged usage and decreased potential of ocular infection, there are proven deleterious side effects with chronic exposure to some preservatives. ${ }^{1}$ These effects have been demonstrated in animals and humans with both in vitro and in vivo models. ${ }^{2-5}$ Studies have also demonstrated that when comparing topical medications with preservatives and their non-preserved counterpart, there is less ocular toxicity, suggesting a direct correlation with the presence of preservative and damage to the ocular surface. ${ }^{5-7}$

One of the more common preservatives used today is benzalkonium chloride (BAK).$^{8}$ BAK is a quaternary ammonium compound whose antimicrobial activity arises from its ability to disrupt cell membranes and potentiate cell death. Along with its antimicrobial activity, BAK is toxic to the corneal epithelium as well as conjunctival epithelium and stroma. ${ }^{4,9-16}$ These changes appear to be both dose- and time-dependent. These toxic side effects come to the forefront in the care of glaucoma patients due to the cumulative amount of BAK that can be introduced through a multiple drop regimen lasting decades. Alternative preservatives have been introduced including oxidizing 
agents, such as sofZia ${ }^{\mathrm{TM}}$ (Alcon Laboratories, Inc., Fort Worth, Texas, USA) that contains borate, zinc and sorbitol. Oxidizing agents may lead to less cell damage than detergent preservatives such as BAK.

This study focuses on the effects of topical antiglaucoma medications in patients with pre-existing ocular surface disease. We aim to quantify changes in tear break-up time (TBUT), corneal staining and the ocular surface disease index (OSDI) after switching from latanoprost with $0.02 \%$ BAK to travoprost with sof: $\mathrm{Zia}^{\mathrm{T}}$.

\section{Materials and methods}

This was a prospective, open label, single-center study involving 40 eyes of twenty consecutive patients presenting to a tertiary care glaucoma practice. The study design was approved by the University of Colorado Internal Review Board. Each patient had been taking latanoprost monotherapy for at least 12 months prior to enrollment. All patients had a baseline tear break-up time (TBUT) of less than 6 seconds. Patients were instructed to avoid adding other topical drops during the study period. Baseline measurements of TBUT, inferior corneal staining and ocular surface disease index (OSDI) were obtained for each enrollee between 7 and 10 AM and in the same examination room at each visit.

TBUT was obtained by placing $5 \mu \mathrm{L}$ of $2 \%$ preservativefree sodium fluorescein $(\mathrm{NaFl})$ to the inferior fornix using a fixed volume micropipette. To thoroughly mix the $\mathrm{NaFl}$ with the tear film, the subject was instructed to blink three

A)

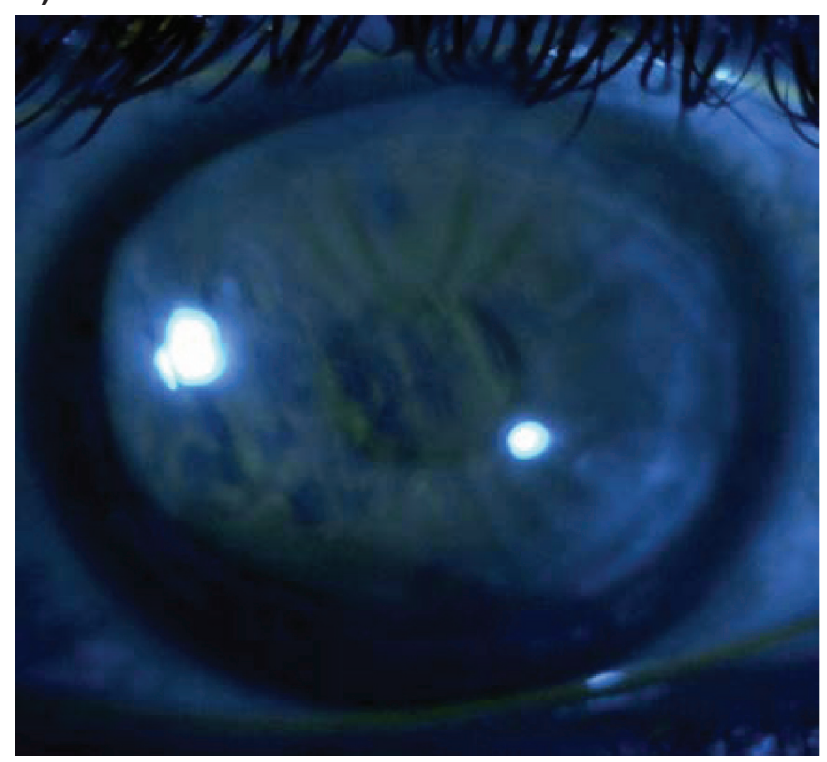

times. The slit lamp was set at a magnification of $16 \times$ using cobalt blue illumination and a stopwatch was used to time the occurrence of the first break in the fluorescein stained tear film (Figure 1A and B). The timer was started immediately after the last blink and stopped at the first break in fluorescein. This was measured three consecutive times and an average of these measurements was used to calculate the final TBUT. At the 8-week follow-up, each TBUT was obtained by the same observer (MYK) using the same method as described above. Inferior corneal staining was measured on a scale of 0 to $4(0=$ none, $1=$ mild, $2=$ moderate, $3=$ severe, $4=$ confluent) at baseline and final follow-up visits. The ocular surface disease index questionnaire was given to the participants at both the initial visit as well as the eight-week follow-up. We used Student's t-test to compare data between the two visits.

\section{Results}

All 20 patients (40 eyes) enrolled in the study who completed the final evaluation were included in data analysis (Figure 2A and B, Table 1). One patient did not return at the 8 -week mark and was excluded from analysis. The patients ranged in age from 42 to 78 years, 16 were Caucasian and 4 African American, 12 were male and all were diagnosed with open angle glaucoma. Prior to starting travoprost, the mean TBUT was $2.02 \pm 0.71$ seconds for the 40 eyes studied. This increased to $6.34 \pm 1.31$ seconds 8 weeks after the switch $(\mathrm{p}<0.001)$. Inferior corneal

\section{B)}

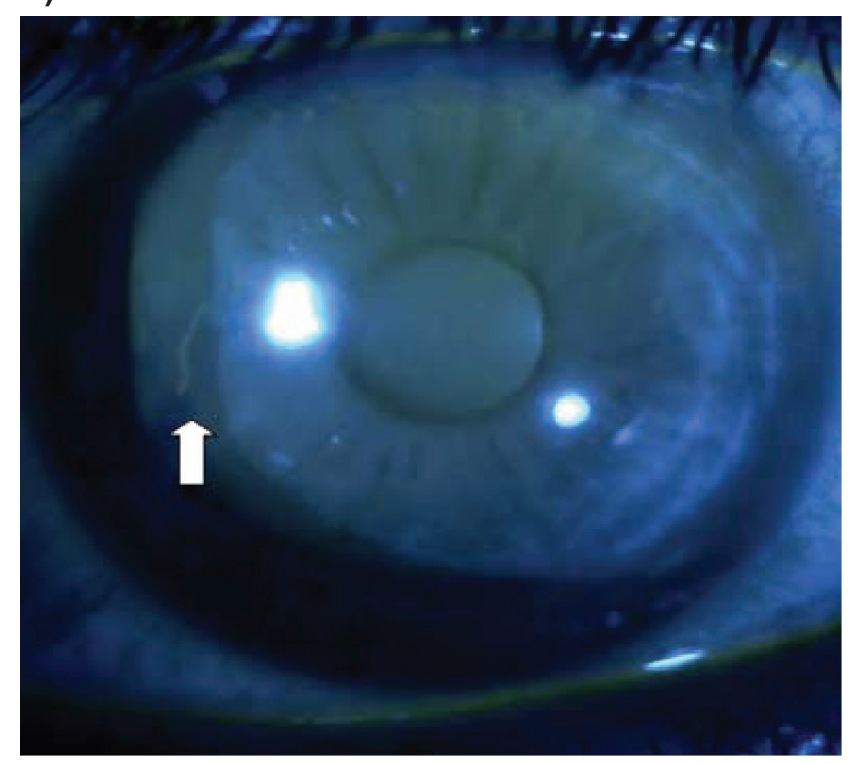

Figure I A) Tear break-up time (TBUT) showing breaks in the fluorescein after I second; B) the same patient on follow-up visit with TBUT (white arrow) first appearing at 7 seconds. 
A)

B)
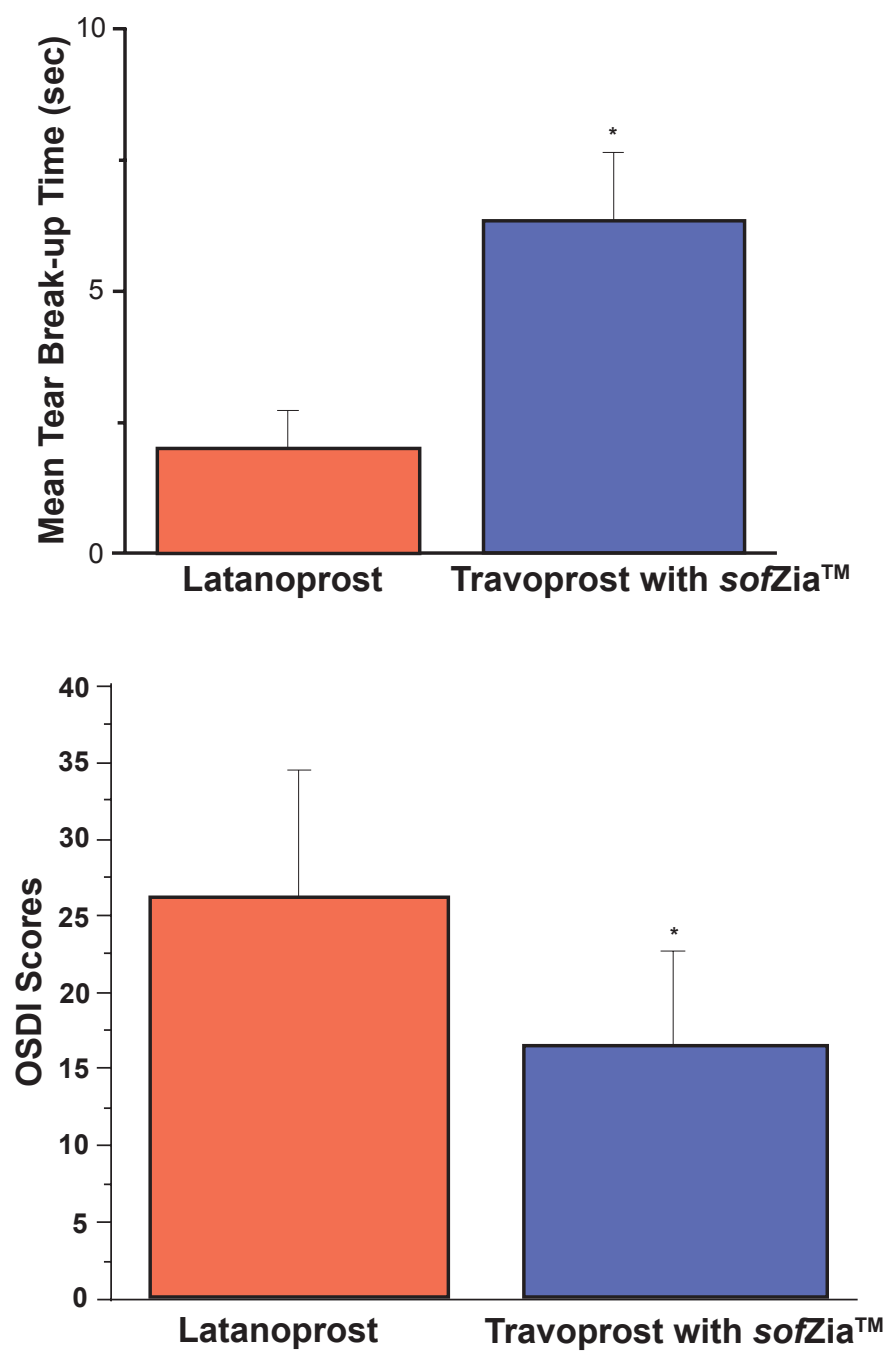

Figure 2 A) Mean tear break-up time on latanoprost and after switch to travoprost without BAK; B) Mean ocular surface disease index on latanoprost and after switch to travoprost without BAK.

Abbreviations: BAK, benzalkonium chloride; OSDI, ocular surface disease index.

staining decreased from $2.40 \pm 0.87$ to $1.38 \pm 0.59$ $(\mathrm{p}<0.001)$. The OSDI initially measured $26.31 \pm 8.25$ which is within the moderate category. Eight weeks after switching to travoprost, the OSDI fell into the mild category: $16.56 \pm 6.19(p<0.001)$ in this same population of 20 patients. There was no statistically significant difference in intraocular pressure (IOP) levels at baseline (14.05 \pm 2.78 $\mathrm{mmHg}$ ) and after the switch to travoprost with sof $\mathrm{Zia}^{\mathrm{TM}}$ $(13.95 \pm 3.04, \mathrm{p}=0.88)$.

\section{Discussion}

BAK is the most commonly used ophthalmic preservative. Multiple studies have delineated the deleterious effects of BAK on conjunctival epithelium, corneal epithelium and corneal stroma. ${ }^{4,-20}$ The microscopic changes seen in patients taking BAK include an increase of pro-inflammatory cytokines, lymphocyte infiltration, fibroblast proliferation, corneal microvilli loss and goblet cell loss in the conjunctival epithelium. In addition, these ocular surface changes may decrease the success rate of glaucoma surgery. ${ }^{21}$ It is also not surprising that changes to the tear film and increase in inflammation result in a worsening of symptoms in patients with preexisting dry eye who are also treated with preserved topical glaucoma drops. ${ }^{14-16}$

Yee et $\mathrm{al}^{22}$ found that there was a marked reduction in toxicity when exposing immortalized human corneal epithelial cells to travoprost with sofZia ${ }^{\text {TM }}$ compared to latanoprost with BAK. Kahook et a ${ }^{19}$ described similar findings in both corneal and conjunctival changes in New Zealand white rabbits. Their results concluded that an increase in inflammation, as measured by lymphocytic infiltration, was found in tissue exposed to latanoprost with BAK as compared to travoprost 
Table I Baseline and follow-up results after switching from latanoprost with BAK to travoprost with sofZia ${ }^{\text {TM }}$

\begin{tabular}{lllc}
\hline & Baseline & Follow-up & p value \\
\hline Corneal staining & $2.40 \pm 0.87$ & $1.38 \pm 0.59$ & $<0.001$ \\
IOP $(\mathrm{mmHg})$ & $14.05 \pm 2.78$ & $13.95 \pm 3.04$ & 0.88 \\
\hline
\end{tabular}

Abbreviations: BAK, benzalkonium chloride; IOP, intraocular pressure.

with SofZia. Also, corneal epithelial damage was statistically higher in the latanoprost with BAK group compared to the travoprost with sofZia ${ }^{\mathrm{TM}}$ group.

The ocular surface disease index, was reported by Schiffman et $\mathrm{al}^{23}$ as both a valid and reliable means to quantify dry eye syndrome. Using this questionnaire, our study found a statistically significant improvement of OSDI in patients switched from latanoprost with BAK to travoprost with sofZia ${ }^{\mathrm{TM}}$. Patients who have less ocular irritation may be more likely to be adherent to their medication regimen. This could lead to more effective lowering of IOP and resultant decrease of glaucomatous vision loss. We also found a statistically significant improvement in tear break-up time (TBUT) after patients were switched to travoprost with sofZia ${ }^{\mathrm{TM}}$. For patients with an already reduced TBUT, this change appears to correlate to a significant improvement in dry eye symptoms as reflected by the improvement in OSDI.

While maintaining antimicrobial activity, studies have shown sofZia ${ }^{\mathrm{TM}}$ to have less deleterious effects than BAK on ocular epithelium in both in vitro and in vivo studies. ${ }^{19,22,24}$ In addition, when comparing travoprost with BAK (Travatan ${ }^{\circledR}$; Alcon Laboratories, Inc., Fort Worth, Texas, USA) to travoprost with sofZia ${ }^{\mathrm{TM}}$ (Travatan $\mathrm{Z}^{\circledR}$; Alcon Laboratories, Inc., Fort Worth, Texas, USA), the IOP-lowering efficacy was found to be identical. ${ }^{25}$ Thus, it appears that BAK is not required for either safety or efficacy of travoprost as an ocular hypotensive agent.

Although the results of both the OSDI and TBUT show a statistically significant improvement when switching from latanoprost with BAK to travoprost with $s o f \mathrm{Zia}^{\mathrm{TM}}$, we focused our study only on patients with measurably decreased TBUT at baseline, thus limiting the generalization of our results. Also, the number of patients enrolled is low and we included both eyes of each patient in our analysis. Finally, all participants lived in the low humidity environment of Denver or surrounding suburbs making generalizations to other geographic areas difficult to make.

The results of this study suggest that a change from a BAK-preserved prostaglandin analog to a prostaglandin analog preserved with sofZia ${ }^{\mathrm{TM}}$ could improve TBUT, corneal staining and OSDI score while maintaining similar IOP-lowering efficacy. These findings cannot be extrapolated to other BAK-preserved medications owing to the limited nature of this open-label pilot study. Further prospective and randomized studies are needed to better understand and confirm the effect of BAK on the ocular surface of glaucoma patients and to quantify possible effects on patient compliance and overall disease progression.

\section{Disclosures}

MYK has received research support and consulting fees from Alcon Laboratories, Inc.

\section{References}

1. Wilcon LA. To preserve or not to preserve, is that the question? Br J Ophthalmol. 1996;80:583-584.

2. De Saint Jean M, Debbasch C, Brignole F, et al. Toxicity of preserved and unpreserved antiglaucoma topical drugs in an in vitro model of conjunctival cells. Curr Eye Res. 2000;20:85-94.

3. Burstein NL. Corneal cytotoxicity of topically applied drugs, vehicles and preservatives. Surv Ophthalmol. 1980;25:15-30.

4. Burstein NL. The effects of topical drugs and preservatives on the tears and corneal epithelium in dry eye. Trans Ophthalmol Soc UK. 1985;104:402-409.

5. De Jong C, Solwijk T, Kuppens E, et al. Topical timolol with and without benzalkonium chloride; epithelial permeability and autofluorescence of the cornea in glaucoma. Graefes Arch Clin Exp Ophthalmol. 1994;232:221-224.

6. Mietz H, Niesen U, Krieglstein GK. The effect of preservatives and antiglaucomatous medication on the histopathology of the conjunctiva. Graefes Arch Clin Exp Ophthalmol. 1994;232:561-565.

7. Pisella PJ, Fillacier K, Elena P, et al. Comparison of the effects of preserved and unpreserved formulations of timolol on the ocular surface of albino rabbits. Ophthalmic Res. 2000;32:3-8.

8. Noecker R. Effects of common ophthalmic preservatives on ocular health. Adv Ther. 2001;18:205-215.

9. Baudouin C, de Lunardo C. Short term comparative study of topical $2 \%$ carteolol with and without benzalkonium chloride in healthy volunteers. Br J Ophthalmol. 1998;82:39-42.

10. Norn MS, Opauszki A. Effects of ophthalmic vehicles on the stability of the precorneal tear film. Acta Ophthalmol. 1977;55:23-34.

11. Yalvaç IS, Gedikoğlu G, Karagöz Y, et al. Effects of antiglaucoma drugs on ocular surface. Acta Ophthalmol Scand. 1995;73:246-248.

12. Kuppens EV, de Jong CA, Stolwijk TR, et al. Effect of timolol with and without preservative on the basal tear turnover in glaucoma. $\mathrm{Br} J \mathrm{Oph}$ thalmol. 1995;79:339-342.

13. Wilson WS, Duncan AJ, Jay JL. Effect of benzalkonium chloride on the stability of the precorneal tear film in rabbit and man. Br J Ophthalmol. 1975;59:667-669.

14. Herreras JM, Pastor JC, Calonge M, et al. Ocular surface alteration after long-term treatment with an antiglaucomatous drug. Ophthalmology. 1992;99:1082-1088.

15. Rolando M, Brezzo V, Giordano G, et al. In: van Bijsterveld OP, Lemp MA, Spinelli D, editors. Symposium on the lacrimal system. Singapore, March 1990. Amsterdam: Kugler and Ghedini; 1991. p. 87-91.

16. Holly FJ. Tear film formation and rupture: an update. In: Holly FJ, ed. The precorneal tear film in health diseases and contact lens wear. Dry Eye Institute Inc., Lubbock; 1986. p. 634-645.

17. Burstein NL. Preservative cytotoxic threshold for benzalkonium chloride and Chlorhexidine digluconate in cat and rabbit corneas. Invest Ophthalmol Vis Sci. 1980;19:308-313. 
18. Marquardt R, Schubert T. Beeinflussung der Tränenfilmaufreisszeit (BUT) durch Betablocker-Augentropfen ohne Konservierungstoffe. Klin Monatsbl Augenheilkd. 1991;199:75-78.

19. Kahook M, Noecker R. Comparison of corneal and conjunctival changes after dosing of travoprost preserved with SofZia, latanoprost with $0.02 \%$ benzalkonium chloride, and preservative-free artificial tears. Cornea. 2008;27:339-343.

20. Noecker RJ, Herrygers LA, Anwaruddin R. Corneal and conjunctival changes caused by commonly used glaucoma medications. Cornea. 2004;23:490-496.

21. Broadway DC, Grierson I, O'Brien C, Hitchings RA. Adverse effects of topical antiglaucoma medication. II. The outcome of filtration surgery. Arch Ophthalmol. 1994;112(11):1446-1454.
22. Yee RW, Norcom EG, Zhao XC. Comparison of the relative toxicity of travoprost $0.004 \%$ without benzalkonium chloride and latanoprost $0.005 \%$ in an immortalized human cornea epithelial cell culture system. Adv Ther. 2006;23:511-519.

23. Schiffman RM, Christianson MD, et al. Reliability and validity of the Ocular Surface Disease Index. Arch Ophthalmol. 2000;118:615-621.

24. Whitson JT, Cavanagh HD, Lakshman N, et al. Assessment of corneal epithelial integrity after exposure to ocular hypotensive agents preserved with and without benzalkonium chloride. Adv Ther. 2006;23:663-671.

25. Lewis RA, Katz GJ, Weiss MJ, et al. Travoprost $0.004 \%$ with and without benzalkonium chloride: a comparison of safety and efficacy. J Glaucoma. 2007;16:98-103 
